\title{
VCG-Kelly Mechanisms for Allocation of Divisible Goods: Adapting VCG Mechanisms to One-Dimensional Signals
}

\author{
Sichao Yang and Bruce Hajek
}

\begin{abstract}
The VCG-Kelly mechanism is proposed, which is obtained by composing the communication efficient, onedimensional signaling idea of Kelly with the VCG mechanism, providing efficient allocation for strategic buyers at Nash equilibrium points. It is shown that the revenue to the seller can be maximized or minimized using a particular one-dimensional family of surrogate valuation functions.
\end{abstract}

Index Terms-

\section{INTRODUCTION}

In this paper, we address the mechanism design problem, which specifies the rules for allocation and payment, for resource allocation in a network with a single network operator and multiple strategic buyers. The network operator, without knowing the valuation functions of the buyers, aims to select an outcome that is efficient, i.e. maximizes the sum of buyers' valuations.

Kelly and his colleagues [1,2] showed that, under the fundamental assumption that all the buyers are price-takers, the optimization problem can be solved in a decentralized way. We call their method the Kelly mechanism. Johari and Tsitsiklis [3] showed that, for strategic buyers using the Kelly mechanism on each of the links, the sum of valuations at the equilibrium point is no less than $75 \%$ of the efficient value. We give two examples which show the undesirable performance of the Kelly mechanism for strategic buyers when each buyer presents a single bid for a path, rather than bids for individual links.

The celebrated Vickrey-Clark-Groves (VCG) mechanism [4-6] is such that true reporting of valuation functions is a dominant strategy equilibrium, but in the context of divisible goods, such as communication rate, the valuation functions are infinite dimensional, and so have prohibitive communication requirements. In [7,8], efficient mechanisms with onedimensional bids are proposed for a single link.

We introduce a mechanism with one dimensional bids for resource allocation in a network in which efficiency is achieved at Nash equilibrium points (NEPs). We call the mechanism the VCG-Kelly mechanism. Although buyers do not have dominant strategies under the VCG-Kelly mechanism

Manuscript received July 1, 2006; revised February 15, 2007.

The authors are with the Department of Electrical and Computer Engineering, and the Coordinated Science Laboratory, University of Illinois at Urbana-Champaign (e-mail: \{syang8,b-hajek\}@uiuc.edu).

Digital Object Identifier 10.1109/JSAC.2007.0708xx. (in contrast to the VCG mechanism), their bids are onedimensional. Simultaneously and independently of our work, Johari and Tsitsiklis [9] developed a family of VCG-like mechanisms using simple scaler bids per player. Their work is more general than ours here, in that their framework involves a convex constraint set and is not limited to network resource allocation settings. However, in the more limited setting of our paper, under a regularity assumption, we find that the payoff function of a buyer is a quasiconcave function of the buyer's bid, that all NEPs are efficient, that the NEP is unique if the efficient allocation is unique, and we characterize the range of possible payments.

Semret [10] proposed an efficient allocation mechanism in which each buyer submits a two dimensional bid, one dimension for unit price, and the other for maximum quantity. Recently, other work using two dimensional bids appeared: $[11,12]$. The mechanism of [12] can be viewed as an instance of the VCG-Kelly mechanism, but for two-parameter surrogate functions. The recent paper of Ausubel [13] describes an allocation mechanism which is a generalization of ascending price auctions, which can provide efficient allocation using one dimensional bids for the allocation problem we address, but the payments are computed by integrating over price trajectories.

The paper is organized as follows. Section II describes the network model, the Kelly mechanism, with either price-taking or strategic buyers (including our two examples), and the VCG mechanism. Section III introduces the VCG-Kelly mechanism and presents the results about it mentioned above. Section IV discusses future work, and proofs are given in the appendix.

\section{System Models}

Consider a network with a finite set of buyers, $R$ and a finite set of links, $J$. The buyers are in one-to-one correspondence with paths, where a path has an associated set of links. We write $j \in r$ if link $j$ is in the path of buyer $r$. The pathlink incidence matrix $A$ is defined by $A_{j r}=1$ if $j \in r$, and $A_{j r}=0$ otherwise. We refer to buyers in gender neutral terms, because in the context of communication networks, they are typically computer processes.

The buyers have elastic demands and the valuation function of a buyer $r$ is represented by $U_{r}\left(x_{r}\right)$, which is an increasing, concave, and continuously differentiable function of its rate allocation $x_{r}$, over $\mathbb{R}_{+}$. In this paper we assume that the valuation functions of the buyers are in monetary units, so 
that if buyer $r$ makes a payment of $m_{r}$ for rate $x_{r}$, the buyer's payoff has the quasilinear form $U\left(x_{r}\right)-m_{r}$. The links have fixed capacities $C_{j}$ for all $j \in J$.

\section{A. System Problem and Efficient Allocations}

A rate allocation vector $\boldsymbol{x}=\left(x_{r}: r \in R\right)$ is called efficient ${ }^{1}$ if it is the solution to the system problem:

System Problem $(\boldsymbol{U}, A, \boldsymbol{C})$

$$
\max _{\boldsymbol{x} \in X_{\boldsymbol{C}}} \sum_{r \in R} U_{r}\left(x_{r}\right)
$$

where $X_{\boldsymbol{C}}=\{\boldsymbol{x}: \boldsymbol{x} \geq 0$ and $A \boldsymbol{x} \leq \boldsymbol{C}\}$.

The system problem is a convex optimization problem with linear constraints, so for any solution $x^{*}$ there is an associated vector $\boldsymbol{\mu}^{*}$ of Lagrange multipliers satisfying the following optimality conditions:

$$
\begin{aligned}
& U_{r}^{\prime}\left(x_{r}^{*}\right) \leq \sum_{j \in r} \mu_{j}^{*}, \text { with equality if } x_{r}^{*}>0, \forall r \in R \\
& \mu_{j}^{*} \geq 0, \quad \text { with equality if } \sum_{r: j \in r} x_{r}^{*}<C_{j}, \quad \forall j \in J
\end{aligned}
$$

Throughout the paper, $X^{*}$ denotes the set of all efficient allocation vectors.

\section{B. Kelly Mechanism}

Kelly and his colleagues [1,2] proposed a decentralized method for solving the system optimization problem. In this section, we briefly describe their method from the mechanism design viewpoint.

In the Kelly mechanism, the communication between any buyer $r$ and the network consists of a one-dimensional bid $w_{r}$ given by buyer $r$ to the network, and a price ${ }^{2} p_{r}$ given by the network to buyer $r$. From the bid $w_{r}$ of buyer $r$, the network constructs a surrogate valuation function $w_{r} \log x_{r}$. The network determines the allocation and payment by maximizing the sum of the surrogate valuations:

Kelly mechanism allocation rule $(A, \boldsymbol{C})$ :

$$
x_{r}^{K}(\boldsymbol{w})=\arg \max _{\boldsymbol{x} \in X_{\boldsymbol{C}}} \sum_{r \in R} w_{r} \log x_{r},
$$

The optimization problem associated with this allocation rule is called the network problem in [2]. To ensure that the mapping is well defined, we require that $x_{r}^{K}(\boldsymbol{w})=0$ if $w_{r}=0$. The solution $\boldsymbol{x}^{K}(\boldsymbol{w})$ can be characterized as follows. It is the vector $\boldsymbol{x} \in X_{\boldsymbol{C}}$ which, together with an associated vector $\boldsymbol{\mu}$ of Lagrange multipliers, satisfies the following conditions.

$$
\begin{gathered}
x_{r}=0, \forall r \in R \text { such that } w_{r}=0 \\
\frac{w_{r}}{x_{r}}=\sum_{j \in r} \mu_{j}, \forall r \in R \text { such that } w_{r}>0 \\
\mu_{j} \geq 0, \text { with equality if } \sum_{r: j \in r} x_{r}<C_{j}, \forall j \in J
\end{gathered}
$$

\footnotetext{
${ }^{1}$ An alternative definition of efficiency, which accounts for the payments of the buyers, is commented on in Section III-B.

${ }^{2}$ In the paper, price always represents "price-per-unit".
}

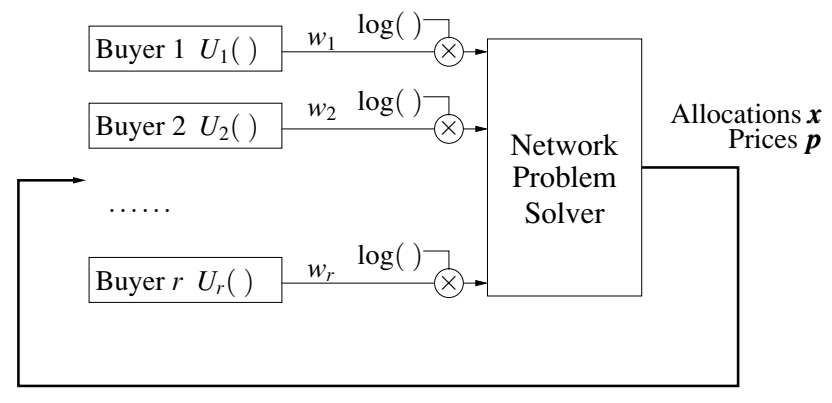

Fig. 1. The Kelly mechanism

Kelly mechanism payment rule $(A, C)$ :

$$
m_{r}^{K}=p_{r} x_{r} \text { where } p_{r}=\sum_{j \in r} \mu_{j} .
$$

Figure 1 depicts the Kelly mechanism. The feedback arrow is not a part of the payment and allocation rules of the mechanism, but is shown to indicate that the equilibrium bid of each buyer depends on the bids of the other buyers.

Next, we discuss two scenarios: the Kelly mechanism applied to price-taking buyers (as in [1,2]) and the Kelly mechanism applied to strategic buyers.

1) Price-taking buyers: A price-taking buyer $r$ sees $p_{r}$ as a fixed price set by the network and it tries to solve the problem of maximizing the payoff function

$$
\Pi_{r}\left(w_{r}\right)=U_{r}\left(\frac{w_{r}}{p_{r}}\right)-w_{r},
$$

which is called the user problem in [2]. It is shown in [2] that, if $\boldsymbol{x}^{*}$ and $\boldsymbol{\mu}^{*}$ satisfy (2) and (3), then for bids $w_{r}=$ $x_{r}^{*} \sum_{j \in r} \mu_{j}^{*}$ and prices $p_{r}^{*}=\sum_{j \in r} \mu_{j}^{*}$, the following is true: $\boldsymbol{x}^{*}$ solves the system problem, and for each $r, w_{r}$ solves the user problem. Thus, the solution to the system problem yields solutions to the user and network problems. The system problem is decomposed into a network problem, which does not involve the valuation functions, and the user problems, which do not involve the network topology.

2) Strategic buyers: A strategic buyer $r$ takes into account how its price is influenced by its bid, $w_{r}$. This induces a game among the buyers, with the payoff for buyer $r$ given by

$$
\Pi_{r}\left(w_{r} ; \boldsymbol{w}_{-r}\right)=U_{r}\left(\frac{w_{r}}{p_{r}(\boldsymbol{w})}\right)-w_{r} .
$$

Next, in two examples, we show that if buyers using the Kelly mechanism are strategic, NEPs may not exist, and if an NEP does exist, the ratio between the sum of valuations at the NEP to the maximum possible sum (the efficiency ratio) can be arbitrarily small ${ }^{3}$.

Consider a network with $L$ links, numbered 1 through $L$, with capacities $C_{l}$. There are $L+1$ buyers, numbered 0 through $L$. The path of buyer zero contains all the links. The path of buyer $l$ for $1 \leq l \leq L$ contains just the single link $l$. We define the rate allocation in the serial network as follows: ${ }^{4}$ If $w=0$, then $\boldsymbol{x}=0$. Otherwise, the network allocates rate $x_{0}$ to buyer

\footnotetext{
${ }^{3}$ These two examples were first presented in [14].

${ }^{4}$ This is a slight modification of the Kelly mechanism defined earlier, because a single-link user bidding zero can sometimes get a nonzero rate, but only if the price on the associated link is zero.
} 


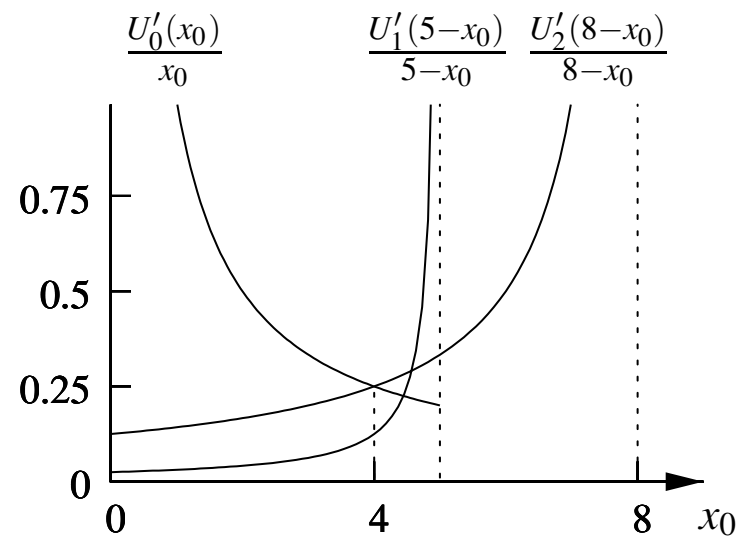

Fig. 2. The uniqueness of $\tilde{\boldsymbol{x}}$

0 and rate $x_{l}=C_{l}-x_{0}$ to each single link buyer, where $x_{0}$ is selected to minimize the sum of the surrogate valuations:

$$
w_{0} \log x_{0}+\sum_{1 \leq l \leq L} w_{l} \log \left(C_{l}-x_{0}\right) .
$$

Example 1: Suppose $L=2$, and $W=\left\{w: x_{i}(w)>0, i=0,1,2\right\}$, or equivalently

$$
\begin{aligned}
W= & \left\{w_{0}>0, w_{1}>0, w_{2}>0\right\} \\
& \cup\left\{\frac{w_{0}}{w_{0}+w_{1}}<\frac{C_{2}}{C_{1}}, w_{0}>0, w_{1}>0, w_{2}=0\right\} \\
& \cup\left\{\frac{w_{0}}{w_{0}+w_{2}}<\frac{C_{1}}{C_{2}}, w_{0}>0, w_{1}=0, w_{2}>0\right\} .
\end{aligned}
$$

It can be shown that all NEPs are in $W$, and there exists a bid vector $\tilde{\boldsymbol{w}}$ in $W$ satisfying the first order necessary condition for an NEP. Let $\tilde{\boldsymbol{x}}=\left(\tilde{x}_{0}, C_{1}-\tilde{x}_{0}, C_{2}-\tilde{x}_{0}\right)$ be the corresponding rate allocation. The value of $\tilde{x}_{0}$ for all such $\tilde{\boldsymbol{w}}$ 's is uniquely defined by

$$
\frac{U_{0}^{\prime}\left(x_{0}\right)}{x_{0}}=\max \left\{\frac{U_{1}^{\prime}\left(C_{1}-x_{0}\right)}{C_{1}-x_{0}}, \frac{U_{2}^{\prime}\left(C_{2}-x_{0}\right)}{C_{2}-x_{0}}\right\}
$$

If the three curves $\frac{U_{0}^{\prime}\left(x_{0}\right)}{x_{0}}, \frac{U_{1}^{\prime}\left(C_{1}-x_{0}\right)}{C_{1}-x_{0}}$ and $\frac{U_{2}^{\prime}\left(C_{2}-x_{0}\right)}{C_{2}-x_{0}}$ do not all meet at a single point, then the value of $\tilde{\boldsymbol{w}}$ is also unique.

Consider the case $C_{1}=5, C_{2}=8, U_{0}(x)=U_{2}(x)=x$, and $U_{1}(x)=\frac{1}{8} x$. There is no triple intersection, $\tilde{\boldsymbol{w}}=(2,0,2)$ is the unique bid vector satisfying the first order necessary condition, and its associated rate vector is $\tilde{\boldsymbol{x}}=(4,1,4)$.

However, $\Pi_{2}\left(\tilde{w}_{0}, \tilde{w}_{1}, 0\right)=3>\Pi_{2}\left(\tilde{w}_{0}, \tilde{w}_{1}, \tilde{w}_{2}\right)=2$ so that $\tilde{\boldsymbol{w}}$ is not an NEP. Also because the point satisfying the first order necessary condition is unique, we conclude that there is no NEP in this example. Intuitively, in this specific case, $C_{1}$ is close to $\tilde{x}_{0}$, so that buyer 2 does not lose much capacity by decreasing its bid from 2 to 0 . Figure 3 shows the payoff function $\Pi_{2}\left(\tilde{w}_{0}, \tilde{w}_{1}, w_{2}\right)$ as a function of $w_{2}$. Note that it is not concave, which helps explain the lack of an NEP.

Example 2: Let $C_{l}=1$ and $U_{l}(x)=x$ for $1 \leq l \leq L$, and $U_{0}(x)=\gamma x$, where $0<\gamma<L$. In this case, the efficient allocation is $x_{0}=0$ and $x_{l}=1$ for $1 \leq l \leq L$. A bid vector $\boldsymbol{w}$ is an NEP if and only if $w_{0}=\left(\frac{\gamma}{\gamma+1}\right)^{2}$ and $\sum_{1 \leq l \leq L} w_{l}=$ $\frac{\gamma}{(\gamma+1)^{2}}$. While the NEP is not unique, all NEPs have the same rate allocation vector

$$
x_{0}=\frac{\gamma}{\gamma+1}, x_{l}=\frac{1}{\gamma+1} \forall 1 \leq l \leq L
$$

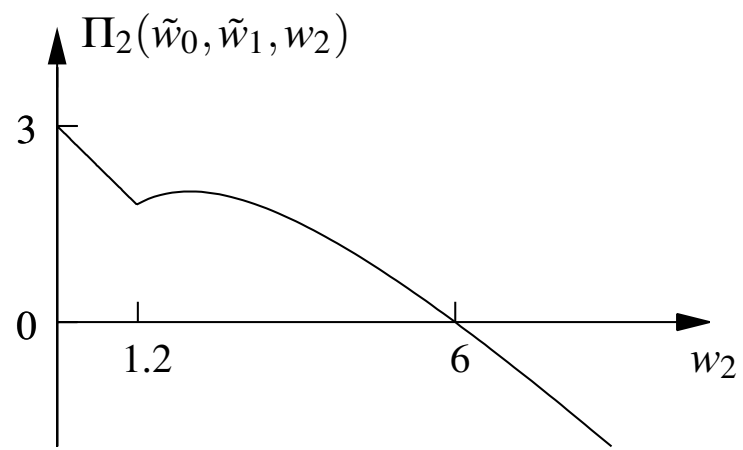

Fig. 3. Nonconcavity of payoff function of buyer 2

Thus, the sum of valuations for the NEP is $\left(\gamma^{2}+L\right) /(\gamma+1)$ and the resulting efficiency ratio for $L \geq \gamma$ is $\left(\gamma^{2}+L\right) /(L(\gamma+1))$. As $L \rightarrow \infty$ the efficiency ratio converges to $1 /(\gamma+1)$. Also, taking $\gamma$ to be an integer and $L=\gamma^{2}$ yields the efficiency ratio $2 /(1+\gamma)$. The ratio converges to zero as $\gamma \rightarrow \infty$. Thus, the loss of efficiency at the NEP can be made arbitrarily close to $100 \%$ by selecting $\gamma$ to be a large enough integer, and $L=\gamma^{2}$.

\section{VCG mechanism}

The VCG mechanism, defined as follows, takes reported valuation functions, $W_{r}(\cdot), r \in R$, as inputs.

VCG mechanism allocation rule $(A, C)$ :

$$
\boldsymbol{x}^{V C G}(\boldsymbol{W})=\arg \max _{\boldsymbol{x} \in X_{\boldsymbol{C}}} \sum_{r \in R} W_{r}\left(x_{r}\right)
$$

VCG mechanism payment rule $(A, C)$ :

$$
\begin{gathered}
m_{r}^{V C G}(\boldsymbol{W})=\max _{\boldsymbol{x} \in X_{\boldsymbol{C}}, x_{r}=0} \sum_{s \in R, s \neq r} W_{s}\left(x_{s}\right) \\
-\sum_{s \in R, s \neq r} W_{s}\left(x_{s}^{V C G}\right)
\end{gathered}
$$

The payment of buyer $r$ in the VCG mechanism is other buyers' maximum surplus with buyer $r$ not present minus the other buyers' surplus with buyer $r$ present. The payoff of buyer $r$ is

$$
\begin{array}{r}
\Pi_{r}\left(x_{r}^{V C G}\right)=\left(U_{r}\left(x_{r}^{V C G}\right)+\sum_{s \in R, s \neq r} W_{s}\left(x_{s}^{V C G}\right)\right) \\
-\max _{x \in X_{\boldsymbol{C}}, x_{r}=0} \sum_{s \in R, s \neq r} W_{s}\left(x_{s}\right)
\end{array}
$$

The last term in (9) does not depend on $x_{r}$. If buyer $r$ reports truthfully, i.e. $W_{r} \equiv U_{r}$, then, by (7), the seller seeks a choice of $x$ that maximizes the sum of the first two terms of the buyer's payoff. Hence, truth reporting is an optimal strategy for buyer $r$, no matter what the other buyers report. All the buyers reporting their true valuations is an NEP for the VCG mechanism, which implies the allocation derived at the NEP of the VCG mechanism is efficient. 


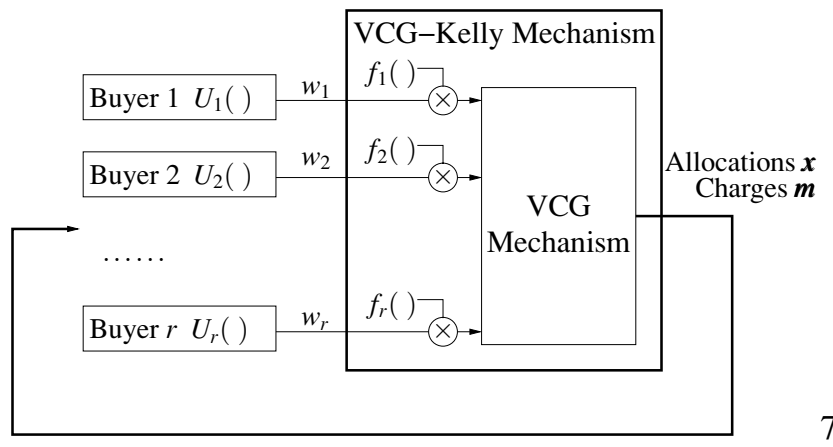

Fig. 4. The VCG-Kelly mechanism

\section{The VCG-Kelly Mechanism}

We propose a new mechanism defined as follows: the buyers send one-dimensional bids and the mechanism forms surrogate valuation functions as in the Kelly mechanism, and then the VCG mechanism is applied to the surrogate valuation functions. We name the mechanism the VCG-Kelly mechanism because it combines the ideas of the VCG mechanism and the Kelly mechanism. See Figure 4.

We introduce a generalization beyond log functions, so that the surrogate functions are $V_{r}\left(w_{r}, x_{r}\right)=w_{r} f_{r}\left(x_{r}\right)$ for $r \in R$, where the $f_{r}$ 's are strictly increasing, strictly concave, and twice differentiable with $f_{r}^{\prime}(0+)=+\infty$ and $f_{r}^{\prime \prime}(x)<0$ over $\mathbb{R}_{+}$. We allow $f_{r}(0)=-\infty$, with the convention that $V_{r}(0,0)=0$. One family of functions from which to select the $f_{r}$ 's is

$$
\phi^{(\alpha)}(x)= \begin{cases}(1-\alpha)^{-1} x^{1-\alpha}, & \text { if } \alpha>0 \text { and } \alpha \neq 1, \\ \log x, & \text { if } \alpha=1,\end{cases}
$$

which is introduced in [15]. The VCG-Kelly mechanism is then defined as follows.

VCG-Kelly mechanism allocation rule $(\boldsymbol{V}, A, \boldsymbol{C})$ :

$$
\boldsymbol{x}^{V C G K}(\boldsymbol{w})=\arg \max _{\boldsymbol{x} \in X_{\boldsymbol{C}}} \sum_{r \in R} V_{r}\left(w_{r}, x_{r}\right)
$$

VCG-Kelly mechanism payment rule $(\boldsymbol{V}, A, \boldsymbol{C})$ :

$$
\begin{array}{r}
m_{r}^{\text {VCGK}}(\boldsymbol{w})=\max _{\boldsymbol{x} \in X_{\boldsymbol{C}}, x_{r}=0} \sum_{s \in R, s \neq r} V_{s}\left(w_{s}, x_{s}\right) \\
-\sum_{s \in R, s \neq r} V_{s}\left(w_{s}, x_{s}^{V C G K}\right)
\end{array}
$$

The rules (11) and (12) are the same as (7) and (8), but with bids $W_{r}\left(x_{r}\right)$ replaced by the surrogate functions $V_{r}\left(w_{r}, x_{r}\right)$, based on one-dimensional bids. The rules $\boldsymbol{x}^{V C G K}$ and $\boldsymbol{m}^{V C G K}$, together with the true valuation functions, $U$, determine a game among the buyers. The payoff of buyer $r$ is

$$
\Pi_{r}\left(w_{r} ; \boldsymbol{w}_{-r}\right)=U_{r}\left(x_{r}^{V C G K}(\boldsymbol{w})\right)-m_{r}^{V C G K}(\boldsymbol{w}) .
$$

A bid vector $\boldsymbol{w}^{N E}$ is an NEP of the game if and only if

$$
\Pi_{r}\left(w_{r}^{N E} ; \boldsymbol{w}_{-r}^{N E}\right) \geq \Pi_{r}\left(w_{r} ; \boldsymbol{w}_{-r}^{N E}\right), \forall w_{r} \geq 0, r \in R .
$$

\section{A. Efficiency of NEPs for the VCG-Kelly mechanism}

The following assumption ensures that each buyer is faced with competition on each link of its path.

Special Buyers Assumption For each link $j$ there are at least two single-link buyers using the link, with valuation functions satisfying $U^{\prime}(0+)=+\infty$. Such buyers are called special. Special buyers participating in the VCG-Kelly mechanism always submit strictly positive bids.

If the special buyers assumption does not hold, there can be many NEPs induced by the VCG-Kelly mechanism that are not efficient. However, instead of using the VCG-Kelly mechanism, one can use an $\epsilon$-relaxation of the VCG-Kelly mechanism, obtained by introducing two fictitious buyers per link, with valuation functions of the form $\epsilon \log (x)$. As $\epsilon \rightarrow \infty$, the payments of the fictitious buyers converge to zero. These issues are explored further in [8] in the case of a single link.

Next we consider the characterizations of efficient allocations and the VCG-Kelly allocation rule under the special buyers assumption. The special buyers assumption insures that all links are saturated for the efficient allocation, so that the optimality conditions (2) and (3) can be simplified. Under the special buyers assumption, $x^{*} \in X_{C}$ is a solution to the system problem (efficient) if and only if there is an associated vector $\boldsymbol{\mu}^{*}$ with nonnegative coordinates, so that

$$
\begin{array}{cc}
U_{r}^{\prime}\left(x_{r}^{*}\right) \leq \sum_{j \in r} \mu_{j}^{*}, \quad \forall r \in R \text { with } x_{r}^{*}=0 \\
U_{r}^{\prime}\left(x_{r}^{*}\right)=\sum_{j \in r} \mu_{j}^{*}, \quad \forall r \in R \text { with } x_{r}^{*}>0 \\
A x^{*}=C
\end{array}
$$

Consider a fixed link $j$. Let $r$ denote one of the special onelink buyers using that link. Then $U_{j}^{\prime}\left(x_{r}^{*}\right)=\mu_{j}$. Thus, although there can be multiple distinct efficient allocation vectors $\boldsymbol{x}^{*}$, for each choice of $\boldsymbol{x}^{*}$, the corresponding vector $\boldsymbol{\mu}^{*}$ is uniquely determined. Moreover, under the special buyers assumption and the assumption that the valuation functions of the buyers are continuously differentiable, the dual problem is strictly convex, so that $\boldsymbol{\mu}^{*}$ does not depend on the choice of $\boldsymbol{x}^{*}$.

Let $\lambda_{r}^{*}=\sum_{j \in r} \mu_{j}^{*}$ for each $r \in R$, or in vector form, $\lambda^{*}=A \boldsymbol{\mu}^{*}$. Then $\lambda_{r}^{*}$ is the price paid by buyer $r$ for the Kelly mechanism with price-taking buyers (denoted by $p_{r}$ in Section II-B.)

We denote the set of special buyers by $R_{s}$, and the space of admissible bid vectors by $W=\left\{w \in \mathbb{R}_{+}^{R}: w_{r}>\right.$ $\left.0 \forall r \in R_{s}\right\}$. Under the special buyers assumption, all links are saturated by $\boldsymbol{x}^{V C G K}(\boldsymbol{w})$ for any $\boldsymbol{w} \in W$. Therefore, $\boldsymbol{x}^{V C G K}(\boldsymbol{w})$ is characterized by the following conditions. For some vector $\mu$, with nonnegative coordinates,

$$
\begin{aligned}
& x_{r}=0, \forall r \in R \text { with } w_{r}=0 \\
& w_{r} f_{r}^{\prime}\left(x_{r}\right)=\sum_{j \in r} \mu_{j}, \quad \forall r \in R \text { with } w_{r}>0 \\
& A \boldsymbol{x}^{*}=\boldsymbol{C}
\end{aligned}
$$

The same observations made for the efficient allocation vectors imply that the choice of $\boldsymbol{\mu}$ in (18) is uniquely determined by $\boldsymbol{w}$, and we can hence denote it as $\boldsymbol{\mu}^{V C G K}(\boldsymbol{w})$. Let $\lambda_{r}^{V C G K}=$ $\sum_{j \in r} \mu_{j}^{V C G K}$ for each $r \in R$, or in vector form, $\lambda^{V C G K}=A \boldsymbol{\mu}^{V C G K}$. 
The following proposition states some regularity properties of the VCG-Kelly mechanism under the special buyers assumption. It also identifies $\lambda_{r}^{V C G K}$ as the marginal price for buyer $r$.

Proposition 3.1: (Regularity of the VCG-Kelly mechanism) Suppose the special buyers assumption holds. The mapping $\boldsymbol{x}^{V C G K}: W \rightarrow X_{C}$ is continuous. For any buyer $r$, and $\boldsymbol{w}_{-r}$ fixed, $\partial x_{r}^{V C G K} / \partial w_{r}>0, \partial \lambda_{r}^{V C G K} / \partial w_{r}>0$, $\left(\partial m_{r}^{V C G K} / \partial w_{r}\right) /\left(\partial x_{r}^{V C G K} / \partial w_{r}\right)=\lambda_{r}^{V C G K}(\boldsymbol{w})$, and the payoff function $\Pi_{r}\left(w_{r}, \boldsymbol{w}_{-r}\right)$, is strictly quasiconcave in $w_{r}$.

Let $W^{N E}$ denote the set of all NEPs for the game, with strategy space $W$ and payoff functions given by (13), induced by the VCG-Kelly mechanism. Our main theorem on the existence and efficiency of NEPs is the following.

Proposition 3.2: Suppose the special buyers assumption holds. The restriction of the mapping $\boldsymbol{x}^{V C G K}$ to $W^{N E}$ is a bijection from $W^{N E}$ to $X^{*}$.

In particular, NEPs exist and correspond to efficient allocations, and if the efficient allocation is unique (for example, if the true valuation functions $U_{r}$ are strictly concave), then the NEP is unique.

\section{B. Prices and revenue under the VCG-Kelly mechanism}

There is considerable flexibility in choosing a VCG-Kelly mechanism, because of the wide range of possible choices of the functions $f_{s}, s \in R$, used to define the surrogate valuation functions. For a given NEP, $\lambda_{r}^{*}$ represents the marginal price for buyer $r$ under the VCG-Kelly mechanism at an NEP, for any choice of the surrogate valuation functions. In contrast, the prices (not the marginal prices), depend heavily on the choice of the surrogate valuation functions. Fix an efficient allocation vector $\boldsymbol{x}^{*}$, and consider a buyer $r$ with $x_{r}^{*}>0$. There is a unique NEP $\boldsymbol{w}^{N E}$ with allocation $\boldsymbol{x}^{*}$, and the price paid by buyer $r$ at that NEP is defined by $p_{r}^{N E}=m_{r}^{V C G K}\left(\boldsymbol{w}^{N E}\right) / x_{r}^{*}$. The next proposition identifies the range of possible prices as the surrogate functions vary.

Proposition 3.3: Suppose the special buyers assumption holds. Fix a choice of the valuation functions $U$, fix an efficient allocation $\boldsymbol{x}^{*}$, and consider a buyer $r$ such that $x_{r}^{*}>0$, Then, under the NEP of the VCG-Kelly mechanisim, $p_{r}^{N E} \in\left(0, \lambda^{*}\right)$. Furthermore, if the function $f_{s}$ used to define the surrogate valuation function for each buyer $s \in R$ is taken to be $\phi^{(\alpha)}$ (defined in (10)), then $\lim _{\alpha \rightarrow+\infty} p_{r}^{N E}=0$ and $\lim _{\alpha \rightarrow 0} p_{r}^{N E}=$ $\lambda_{r}^{*}$.

Corollary 3.4: The total revenue of the seller is in the interval $\left(0, \sum_{j \in J} \mu_{j}^{*} C_{j}\right)$. If the function $f_{r}$ for each buyer $r$ is taken to be $\phi^{(\alpha)}$, then the total revenue converges to zero as $\alpha \rightarrow+\infty$, and to $\sum_{j \in J} \mu_{j}^{*} C_{j}$ as $\alpha \rightarrow 0$.

We remark that an alternative definition of efficiency in the context of mechanism design is to find an allocation and payment rule so that, at the NEP, the sum of the payoff functions of the buyers is maximized. That is, such definition includes the payments of the buyers. Proposition 3.3 shows that the VCG-Kelly mechanism, using functions $f_{s}$ of the form $\phi^{(\alpha)}$, is asymptotically efficient in this alternative sense, in the limit as $\alpha \rightarrow \infty$, because the allocation maximizes the sum of the valuation functions, while the sum of payments converges to zero.

\section{FUTURE WORK}

Future work includes exploring the space of surrogate valuation functions further, investigation of dynamics for arriving at NEPs, application to other contexts, and implementation issues, such as interaction with existing network protocols, such as TCP.

\section{APPENDIX}

The proofs of the propositions in this paper, including lemmas, are given in this appendix.

Lemma 1.1: The mapping $\boldsymbol{x}^{\text {VCGK }}: W \rightarrow X_{C}$ is continuous.

Proof: Suppose $\boldsymbol{w}^{(n)} \rightarrow \overline{\boldsymbol{w}}$ as $n \rightarrow \infty$, such that $\boldsymbol{w}^{(n)} \in$ $W$ for all $n$ and $\overline{\boldsymbol{w}} \in W$. Let $\boldsymbol{x}^{(n)}=\boldsymbol{x}^{V C G K}\left(\boldsymbol{w}^{(n)}\right)$ for each $n$. It must be shown that $\boldsymbol{x}^{(n)} \rightarrow \boldsymbol{x}^{V C G K}(\overline{\boldsymbol{w}})$. Since $X_{\boldsymbol{C}}$ is compact, we assume $\boldsymbol{x}^{(n)} \rightarrow \widetilde{\boldsymbol{x}}$ for some $\widetilde{\boldsymbol{x}} \in X_{\boldsymbol{C}}$, and then it suffices to show that $\widetilde{\boldsymbol{x}}=\boldsymbol{x}^{V C G K}(\overline{\boldsymbol{w}})$. Let $\Phi$ denote the function on $W \times X_{\boldsymbol{C}}$ defined by $\Phi(\boldsymbol{w}, \boldsymbol{x})=\sum_{r \in R} V_{r}\left(w_{r}, x_{r}\right)$. Let $\widehat{\boldsymbol{x}} \in X_{\boldsymbol{C}}$ and $\epsilon>0$ be arbitrary. By perturbing $\widehat{x}$ slightly, we can obtain $\widehat{x}^{\epsilon} \in X_{C}$, that has strictly positive coordinates, and satisfies $\Phi(\overline{\boldsymbol{w}}, \widehat{\boldsymbol{x}}) \leq \Phi\left(\overline{\boldsymbol{w}}, \widehat{\boldsymbol{x}}^{\epsilon}\right)+\epsilon$. We have

$$
\begin{aligned}
\Phi(\overline{\boldsymbol{w}}, \widehat{\boldsymbol{x}}) & \leq \Phi\left(\overline{\boldsymbol{w}}, \widehat{\boldsymbol{x}}^{\epsilon}\right)+\epsilon=\lim _{n \rightarrow \infty} \Phi\left(\boldsymbol{w}^{(n)}, \widehat{\boldsymbol{x}}^{\epsilon}\right)+\epsilon \\
& \leq \lim \sup _{n \rightarrow \infty} \Phi\left(\boldsymbol{w}^{(n)}, \boldsymbol{x}^{(n)}\right)+\epsilon \\
& \leq \Phi(\overline{\boldsymbol{w}}, \widetilde{\boldsymbol{x}})+\epsilon,
\end{aligned}
$$

where the last inequality is a consequence of the fact that $\Phi$ is upper semicontinuous. Since $\epsilon$ is arbitrary, if follows that $\Phi(\overline{\boldsymbol{w}}, \widehat{\boldsymbol{x}}) \leq \Phi(\overline{\boldsymbol{w}}, \widetilde{\boldsymbol{x}})$. Since $\widehat{\boldsymbol{x}} \in X_{\boldsymbol{C}}$ is arbitrary, it follows that $\widetilde{\boldsymbol{x}}=\boldsymbol{x}^{V C G K}(\overline{\boldsymbol{w}})$, which completes the proof of the lemma.

Lemma 1.2: Let $I$ be any subset of $R$ which includes the special buyers. Then for $w_{r} \equiv 0$ for $r \in W-I$, both $\boldsymbol{x}^{V C G K}(\boldsymbol{w})$ and $\boldsymbol{\mu}^{V C G K}(\boldsymbol{w})$ are continuously differentiable functions of $\left(w_{r}\right.$ : $r \in I)$ over the set $(0,+\infty)^{I}$.

Proof: The proof is given for the special case $I=$ $R$, which is sufficient, because buyers in $R-I$ can be ignored. The optimality conditions (17)-(19) can be written as $F\left(\boldsymbol{x}^{V C G K}, \boldsymbol{\mu}^{V C G K}, \boldsymbol{w}\right)=0$, where

$$
F(\boldsymbol{x}, \boldsymbol{\mu}, \boldsymbol{w})=\left(\begin{array}{ll}
w_{r} f_{r}^{\prime}\left(x_{r}\right)-\sum_{j \in r} \mu_{j}, & r \in R \\
\sum_{r: j \in r} x_{r}-C_{j}, & j \in J
\end{array}\right) .
$$

The function $F$ is continuously differentiable and

$$
-\left.\left(\frac{\partial F}{\partial \boldsymbol{x}} \frac{\partial F}{\partial \boldsymbol{\mu}}\right)\right|_{\boldsymbol{w}}=\left(\begin{array}{cc}
D & A^{T} \\
-A & 0
\end{array}\right),
$$

where $D=-\operatorname{diag}\left(w_{r} f_{r}^{\prime \prime}\left(x_{r}^{V C G K}\right)\right)$. Obviously, $D$ is positive definite. For any $J$-dimensional vector $\alpha \neq \mathbf{0}, A^{T} \alpha \neq \mathbf{0}$ because $A$ has full rank (because of the special buyers assumption). Then, $\alpha^{T} A D^{-1} A^{T} \alpha=\left(A^{T} \alpha\right)^{T} D^{-1}\left(A^{T} \alpha\right)>0$, because $D$ is positive definite. Hence, $A D^{-1} A^{T}$ is positive definite. Thus, the matrix $Q$ defined by $Q=\left(A D^{-1} A^{T}\right)^{-1}$ exists and is also positive definite. It is then straightforward to check that the matrix on the right hand side of (20) is nonsingular, and its inverse is:

$$
\left(\begin{array}{cc}
H & -D^{-1} A^{T} Q \\
Q A D^{-1} & Q
\end{array}\right)
$$


where $H=D^{-1}-D^{-1} A^{T} Q A D^{-1}$. Therefore, by the implicit function theorem [16], the functions $\boldsymbol{x}^{V C G K}(\boldsymbol{w})$ and $\boldsymbol{\mu}^{V C G K}(\boldsymbol{w})$ are continuously differentiable, and

$$
\left(\begin{array}{c}
\frac{\partial \boldsymbol{x}^{V C G K}}{\partial \boldsymbol{w}} \\
\frac{\partial \boldsymbol{\mu}^{V C G K}}{\partial \boldsymbol{w}}
\end{array}\right)=-\left.\left(\frac{\partial F}{\partial \boldsymbol{x}} \frac{\partial F}{\partial \boldsymbol{\mu}}\right)^{-1}\right|_{\boldsymbol{w}} \cdot \frac{\partial F}{\partial \boldsymbol{w}}
$$

or

$$
\begin{aligned}
& \frac{\partial \boldsymbol{x}^{V C G K}}{\partial \boldsymbol{w}}=H \operatorname{diag}\left(f_{r}^{\prime}\right) \\
& \frac{\partial \boldsymbol{\mu}^{V C G K}}{\partial \boldsymbol{w}}=Q A D^{-1} \operatorname{diag}\left(f_{r}^{\prime}\right) .
\end{aligned}
$$

Lemma 1.3: Fix a buyer $r \in R$ and the other buyers' bids $\boldsymbol{w}_{-r}$. Then the partial derivatives of $x_{r}^{V C G K}$ and $\lambda_{r}^{V C G K}$ with respect to $w_{r}$ are strictly positive.

Proof: Since $\boldsymbol{\lambda}^{V C G K}=A^{T} \boldsymbol{\mu}^{V C G K}$,

$$
\frac{\partial \boldsymbol{\lambda}^{V C G K}}{\partial \boldsymbol{w}}=A^{T} Q A D^{-1} \operatorname{diag}\left(f_{r}^{\prime}\right) .
$$

Let $\boldsymbol{e}_{r}=(0, \cdots, 1, \cdots, 0)^{T}$ be the elementary vector with $r$-th entry 1 . Then, the $r$-th diagonal entry of $A^{T} Q A$ satisfies $\boldsymbol{e}_{r}^{T} A^{T} Q A \boldsymbol{e}_{r}>0$, because $A \boldsymbol{e}_{r} \neq 0$ and $Q$ is positive definite. Notice that, in (23), the diagonal elements in $\frac{\partial \boldsymbol{\lambda}^{\text {VCGK }}}{\partial \boldsymbol{w}}$ have the same sign as the diagonal elements in $A^{T} Q A$ because $D^{-1} \operatorname{diag}\left(f_{r}^{\prime}\right)$ is a positive definite diagnal matrix. Hence, $\frac{\partial \lambda_{r}^{V C G K}}{\partial w_{r}}>0$ for any $r \in R$.

Let $\mathcal{R}(B)$ denote the range of a matrix $B$ and $\mathcal{N}(B)$ the null space of $B$. We claim that multiplication by the symmetric matrix $G=D^{-\frac{1}{2}} A^{T} Q A D^{-\frac{1}{2}}$ is equivalent to orthogonal projection onto $\mathcal{R}\left(D^{-\frac{1}{2}} A^{T}\right)$. Indeed, on one hand if $\beta \in$ $\mathcal{R}\left(D^{-\frac{1}{2}} A^{T}\right)$ then $\beta=D^{-\frac{1}{2}} A^{T} \alpha$ for some vector $\alpha$, and $G \beta=\beta$, and on the other hand if $\beta \perp \mathcal{R}\left(D^{-\frac{1}{2}} A^{T}\right)$ then $\beta \in$ $\mathcal{N}\left(A D^{-\frac{1}{2}}\right)$ and $G \beta=0$. Therefore, multiplication by $I-G$ is equivalent to orthogonal projection onto $\mathcal{R}^{\perp}\left(D^{-\frac{1}{2}} A^{T}\right)$. Thus $u^{T}\{I-G\} u \geq 0$ for any vector $u \in \mathbb{R}^{R}$ with equality if and only if $u \in \mathcal{R}\left(D^{-\frac{1}{2}} A^{T}\right)$. Therefore $v^{T} H v \geq 0$ for any vector $v \in \mathbb{R}^{R}$ with equality if and only if $v \in \mathcal{R}\left(A^{T}\right)$. The special buyers assumption implies that for an appropriate ordering of the links, $A$ has the structure $A=\left(I_{J \times J}: I_{J \times J}: \widetilde{A}\right)$, which implies that $e_{r} \notin \mathcal{R}\left(A^{T}\right)$. It follows that the diagonal entries of the matrix $H$ are strictly positive, so that $\frac{\partial x_{r}^{V C G K}}{\partial w_{r}}>0$ for any $r \in R$.

Remark 1.1: For any rate allocation $x$ which saturates all the links and arbitrary vector of Lagrange multipliers $\mu$, and vector $\lambda$ defined by $\lambda=A \mu$,

$$
\sum_{s \in R} \lambda_{s} x_{s}=\sum_{s \in R} \sum_{j \in s} \mu_{j} x_{s}=\sum_{j \in J} \mu_{j} \sum_{s: j \in s} x_{s}=\sum_{j \in J} \mu_{j} C_{j}
$$

Therefore, if $\overline{\boldsymbol{x}}$ and $\boldsymbol{x}$ are two rate allocation vectors that both saturate all the links,

$$
\sum_{s \in R} \lambda_{s}\left(\bar{x}_{s}-x_{s}\right)=0 .
$$

Lemma 1.4: For any buyer $r$ and $\boldsymbol{w}_{-r}$ fixed, $\left(\partial m_{r}^{V C G K} / \partial w_{r}\right) /\left(\partial x_{r}^{V C G K} / \partial w_{r}\right)=\lambda_{r}^{V C G K}(\boldsymbol{w})$, and the payoff function $\Pi_{r}\left(w_{r}, \boldsymbol{w}_{-r}\right)$ of buyer $r$ is strictly quasiconcave in $w_{r}$. A vector $\boldsymbol{w} \in W$ is an NEP if and only if the following conditions hold:

$$
\begin{aligned}
& U_{r}^{\prime}\left(x_{r}^{V C G K}\left(w_{r} ; \boldsymbol{w}_{-r}\right)\right)=\sum_{j \in r} \mu_{j}^{V C G K}\left(w_{r} ; \boldsymbol{w}_{-r}\right), \quad \text { if } w_{r}>0, \\
& U_{r}^{\prime}(0) \leq \sum_{j \in r} \mu_{j}^{V C G K}\left(0 ; \boldsymbol{w}_{-r}\right), \quad \text { if } w_{r}=0 .
\end{aligned}
$$

Proof: The payoff function $\Pi_{r}\left(w_{r} ; \boldsymbol{w}_{-r}\right)$, which has the form (13), is a continuously differentiable function of $w_{r}$ by Lemma 1.2. The partial derivative of $m_{r}^{V C G K}\left(w_{r} ; \boldsymbol{w}_{-r}\right)$ with respect to bid $w_{r}$ is

$$
\begin{aligned}
\frac{\partial m_{r}^{V C G K}}{\partial w_{r}}\left(w_{r} ; \boldsymbol{w}_{-r}\right) & =-\sum_{s \neq r} w_{s} f_{s}^{\prime}\left(x_{s}^{V C G K}(\boldsymbol{w})\right) \frac{\partial x_{s}^{V C G K}}{\partial w_{r}}(\boldsymbol{w}) \\
& =-\sum_{s \neq r} \lambda_{s}^{V C G K}(\boldsymbol{w}) \frac{\partial x_{s}^{V C G K}}{\partial w_{r}}(\boldsymbol{w}) \\
& =\lambda_{r}^{V C G K}(\boldsymbol{w}) \frac{\partial x_{r}^{V C G K}}{\partial w_{r}}(\boldsymbol{w}) .
\end{aligned}
$$

The last equaliy above holds by (24). The first part of the lemma, identifying $\lambda_{r}^{V C G K}(\boldsymbol{w})$ as the marginal price, is proven by (25). Also,

$\frac{\partial \prod_{r}}{\partial w_{r}}\left(w_{r} ; \boldsymbol{w}_{-r}\right)=\frac{\partial x_{r}^{V C G K}}{\partial w_{r}}(\boldsymbol{w})\left\{U_{r}^{\prime}\left(x_{r}^{V C G K}(\boldsymbol{w})\right)-\lambda_{r}^{V C G K}(\boldsymbol{w})\right\}$.

By Lemma $1.3, \frac{\partial x_{r}^{V C G K}}{\partial w_{r}}>0$ and $U_{r}^{\prime}\left(x_{r}^{V C G K}(\boldsymbol{w})\right)-\lambda_{r}^{V C G K}(\boldsymbol{w})$ is a strictly decreasing function of $w_{r}$. Therefore, the payoff $\Pi_{r}\left(w_{r} ; \boldsymbol{w}_{-r}\right)$ is a strictly quasiconcave function of $w_{r}$. For other bids $\boldsymbol{w}_{-s}$ fixed, $w_{r}$ is the best response of the buyer $r$ if and only if the conditions given in the lemma hold.

Proof of Proposition 3.1 Lemmas 1.1, 1.3, 1.4, and establish the the proposition.

Proof of Proposition 3.2 It is shown that (i) $\boldsymbol{x}^{V C G K}$ maps $W^{N E}$ into $X^{*}$, (ii) the mapping is surjective, and (iii) the mapping is injective. If $w$ is an NEP, the conditions of Lemma 1.4 hold. Because $x_{r}^{V C G K}(\boldsymbol{w})=0$ if and only if $w_{r}=0$, the vectors $\boldsymbol{x}^{V C G K}$ and $\boldsymbol{\mu}^{V C G K}$ satisfy the efficiency conditions (14)(16). Therefore, $\boldsymbol{x}^{V C G K}(\boldsymbol{w})$ is efficient, so that $\boldsymbol{x}^{V C G K}$ maps $W^{N E}$ into $X^{*}$.

Fix an efficient allocation $x^{*}$. The optimality conditions (14)-(16) are satisfied, for a vector $\mu^{*}$ uniquely determined by $\boldsymbol{x}^{*}$. Let $w_{r}=0$ if $x_{r}^{*}=0$, and $w_{r}=\sum_{j \in r} \mu_{j} / f_{r}^{\prime}\left(x_{r}^{*}\right)$ if $x_{r}>0$. Then conditions (17)-(19) are satisfied, so that $\boldsymbol{x}^{V C G K}(\boldsymbol{w})=\boldsymbol{x}^{*}$ and $\boldsymbol{\mu}^{V C G K}(\boldsymbol{w})=\boldsymbol{\mu}^{*}$. Conditions (14)-(16) and (17)-(19) imply that the conditions of Lemma 1.4 are also satisfied, so that $\boldsymbol{w}$ is an NEP. The required mapping is surjective.

Fix an efficient allocation $x^{*}$ and suppose $x^{V C G K}(\boldsymbol{w})=x^{*}$ for some NEP $\boldsymbol{w}$. Then $x^{*}$ and the associated Lagrange multipliers $\boldsymbol{\mu}^{V C G K}(w)$ satisfy the conditions of Lemma 1.4 , and the conditions (17)-(19). The conditions of Lemma 1.4, under the special buyers assumption, show that $\boldsymbol{\mu}^{V C G K}(\boldsymbol{w})$ is completely determined by the choice of $\boldsymbol{x}^{*}$, and then conditions (17)-(19) show that $\boldsymbol{w}$ is also uniquely determined by the choice of $\boldsymbol{x}^{*}$. So the required mapping is also injective, so that it is bijective, as was to be proved.

Proof of Proposition 3.3 Due to the special buyers assumption, any allocation given up by buyer $r$ has positive 
value for some other buyer, so that $m_{r}^{V C G K}\left(\boldsymbol{w}^{N E}\right)>0$, and therefore $p_{r}^{N E}>0$. Motivated by the definition of the payment function $m_{r}^{V C G K}$ in (12), we let

$$
\overline{\boldsymbol{x}}=\underset{\boldsymbol{x} \in X_{\boldsymbol{C}}, x_{r}=0}{\operatorname{argmax}} \sum_{s \in R, s \neq r} w_{s}^{N E} f_{s}\left(x_{s}\right),
$$

The special buyers assumption implies that $x^{*}$ and $\bar{x}$ both saturate all the links, so by Remark 1.1,

$$
\sum_{s \in R, s \neq r} \lambda_{s}^{*}\left(\bar{x}_{s}-x_{s}^{*}\right)=\lambda_{r}^{*} x_{r}^{*}
$$

Comparing (15) and (18) shows that $w_{s}^{N E} f_{s}^{\prime}\left(x_{s}^{*}\right)=\lambda_{s}^{*}$ for all $s$ such that $w_{s}>0$. This, the fact the functions $f_{s}$ are strictly concave, the fact $\bar{x}_{s} \neq x_{s}^{*}$ for at least one $s \neq r$, and (27) yield:

$$
\begin{aligned}
m_{r}^{V G G K}\left(\boldsymbol{w}^{N E}\right) & =\sum_{s \in R, s \neq r} w_{s}^{N E}\left(f_{s}\left(\bar{x}_{s}\right)-f_{s}\left(x_{s}^{*}\right)\right) \\
& =\sum_{s \in R, s \neq r} \frac{\lambda_{s}^{*}\left(f_{s}\left(\bar{x}_{s}\right)-f_{s}\left(x_{s}^{*}\right)\right)}{f_{s}^{\prime}\left(x_{s}^{*}\right)} \\
& <\sum_{s \in R, s \neq r} \lambda_{s}^{*}\left(\bar{x}_{s}-x_{s}^{*}\right) \\
& =\lambda_{r}^{*} x_{r}^{*}
\end{aligned}
$$

Thus $p_{r}^{N E}<\lambda_{r}^{*}$. So we've proved $p_{r}^{N E} \in\left(0, \lambda_{r}^{*}\right)$.

Next, suppose that $f_{s}\left(x_{s}\right)=\phi^{(\alpha)}\left(x_{s}\right)$ for all $s$, were $\alpha>0$. Then (28) becomes

$$
m_{r}^{V C G K}\left(\alpha, \boldsymbol{w}^{N E}(\alpha)\right)=\frac{1}{1-\alpha} \sum_{s \in R, s \neq r} \lambda_{s}^{*}\left(\bar{x}_{s}\left(\frac{x_{s}^{*}}{\bar{x}_{s}}\right)^{\alpha}-x_{s}^{*}\right)
$$

If $\alpha>1$ then $1-\alpha<0$, and (29) yields

$$
m_{r}^{V C G K}\left(\alpha, \boldsymbol{w}^{N E}(\alpha)\right) \leq \frac{1}{\alpha-1} \sum_{s \in R, s \neq r} \lambda_{s}^{*} x_{s}^{*} \text { if } \alpha>1 .
$$

Therefore, $\lim _{\alpha \rightarrow \infty} m_{r}\left(\alpha, \boldsymbol{w}^{N E}(\alpha)\right)=0$.

By (27) and (29),

$$
\begin{aligned}
& m_{r}^{V C G K}\left(\alpha, \boldsymbol{w}^{N E}(\alpha)\right) \\
& =\frac{1}{1-\alpha}\left\{\lambda_{r}^{*} x_{r}^{*}+\sum_{s \in R, s \neq r} \lambda_{s}^{*} \bar{x}_{s}\left(\left(\frac{x_{s}^{*}}{\bar{x}_{s}}\right)^{\alpha}-1\right)\right\}
\end{aligned}
$$

So, $\lim _{\alpha \rightarrow 0} m_{r}^{V C G K}\left(\alpha, \boldsymbol{w}^{N E}(\alpha)\right)=\lambda_{r}^{*} x_{r}^{*}$, which implies that $\lim _{\alpha \rightarrow 0} p_{r}^{N E}=\lambda_{r}^{*}$.

\section{ACKNOWLEDGEMENT}

The authors wish to thank Prof. Steven R. Williams and the reviewers for their useful comments. This work was supported in part by the National Science Foundation under grants NSF CNS 05-19691 and NSF ECS 06-21416.

\section{REFERENCES}

[1] F. P. Kelly, "Charging and rate control for elastic traffic," European Transactions on Telecommunications, vol. 8, pp. 33-37, 1997.
[2] F. P. Kelly, A. K. Maulloo, and D. Tan, "Rate control in communication networks:shadow prices, proportional fairness and stability," Journal of the Operational Research Society, vol. 49, pp. 237-252, 1998.

[3] R. Johari and J. N. Tsitsiklis, "Efficiency loss in network resource allocation game," Mathematics of Operations Research, vol. 29, no. 3, pp. 407-435, 2004.

[4] W. Vickrey, "Counterspeculation, auctions and competitive sealed tenders," Journal of Finance, vol. 16, no. 1, pp. 8-37, 1961.

[5] E. Clark, "Multipart pricing of public goods," Public Choice, vol. 2, pp. 19-33, 1971.

[6] T. Groves, "Incentives in teams," Econometrica, vol. 41, no. 4, pp. 617631, 1973.

[7] R. T. Maheswaran and T. Başar, "Social welfare of selfish agents; motivating efficiency for divisible resources," in Proceedings of 43rd IEEE Conference on Decision and Control, (Bahamas), December 2004.

[8] S. Yang and B. Hajek, "Revenue and stablity of a mechanism for efficient allocation of a divisible good," Preprint, 2005.

[9] R. Johari and J. N. Tsitsiklis, "Communication requirements of VCGlike mechanisms in convex environments," in Proceedings of Allerton Conference, 2005.

[10] N. Semret, Market mechanisms for network resource sharing. PhD thesis, Columbia University, 1999.

[11] T. Stoenescu and J. Ledyard, "Implementation in nash equilibria of a rate allocation problem in networks," in Proceedings of the 44th Annual Allerton Conference on Communications, Control, and Computing, September 2006.

[12] A. Dimakis, R. Jain, and J. Walrand, "Mechanisms for efficient allocation in divisible capacity networks," in Proceedings of the 45th IEEE Conference on Decision and Control, December 2006.

[13] L. Ausubel, "An efficient dynamic auction for heterogeneous commodities," The American Economic Review, vol. 96, pp. 602-629, 2006.

[14] B. Hajek and S. Yang, "Strategic buyers in a sum bid game for flat networks," in IMA Workshop 6: Control and Pricing in Communication and Power Networks, (University of Minnesota), March 2004.

[15] J. Mo and J. Walrand, "Fair end-to-end window-based congestion control," IEEE/ACM Transactions on Networking, vol. 8, pp. 556-567, October 2000.

[16] D. P. Bertsekas, Nonlinear Programming. Athena Scientific, 2 ed., 1999.

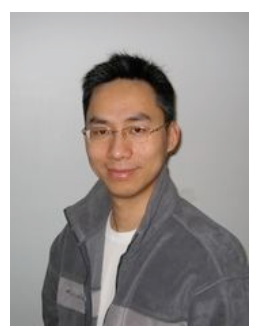

Sichao Yang received the B.E. and M.E. degrees in Electronic Engineering from Shanghai Jiao Tong University. Since 2001 he has been with the University of Illinois at Urbana-Champaign, where he obtained the M.S. degree in mathematics and is currently pursuing the $\mathrm{Ph} . \mathrm{D}$. degree in electrical and computer engineering. His research interests include communication and computer networks, mechanism design and auction algorithms, combinatorial optimization.

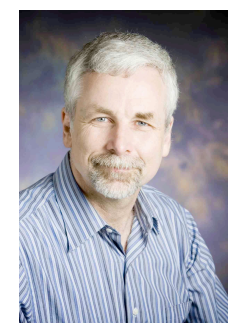

Bruce Hajek (M'79-SM'84-F'89) received a B.S. in Mathematics and an M.S. in Electrical Engineering from the University of Illinois at Urbana-Champaign in 1976 and 1977, and a Ph. D. in Electrical Engineering from the University of California at Berkeley in 1979. He is a Professor in the Department of Electrical and Computer Engineering and in the Coordinated Science Laboratory at the University of Illinois at Urbana-Champaign, where he has been since 1979. His research interests include communication and computer networks, stochastic systems, combinatorial and nonlinear optimization, and information theory. He served as Associate Editor for Communication Networks and Computer Networks for the IEEE Transactions on Information Theory (1985-1988), as Editor-inChief of the same Transactions (1989-1992), and as President of the IEEE Information Theory Society (1995).

Dr. Hajek was a winner of the 1973 USA Mathematical Olympiad, and he received the Eckman Award of the American Automatic Control Council(1982), an NSF Presidential Young Investigators Award (1984), an Outstanding Paper Award from the IEEE Control Systems Society (1985). $\mathrm{He}$ is a member of the US National Academy of Engineering and he received the 2003 IEEE Kobayashi Computer Communications Award. 